\title{
Stress-Transfer in Anisotropic and Environmentally Adaptive Cellulose Whisker Nanocomposites
}

\author{
Rafeadah Rusli, ${ }^{\dagger}$ Kadhiravan Shanmuganathan, ${ }^{\ddagger}$ Stuart J. Rowan, ${ }^{\ddagger}$ Christoph Weder, ${ }^{\ddagger, \S}$ \\ and Stephen J. Eichhorn*, ${ }^{*}$
}

\author{
Materials Science Centre and the Northwest Composites Centre, School of Materials, University of \\ Manchester, Grosvenor Street, Manchester, M1 7HS, United Kingdom, Department of Macromolecular \\ Science and Engineering, Kent Hale Smith Building, Case Western Reserve University, 2100 Adelbert \\ Road, Cleveland, Ohio 44106, and Adolphe Merkle Institute and Fribourg Center for Nanomaterials, \\ University of Fribourg, $\mathrm{CH}-1700$ Fribourg, Switzerland
}

\begin{abstract}
Quantitative insights into the stress-transfer mechanisms that determine the mechanical properties of tunicate cellulose whisker/poly(vinyl acetate) nanocomposites were gained by Raman spectroscopy. The extent of stresstransfer is influenced by local orientation (or anisotropy) of the whiskers, which in turn is governed by the processing conditions used to fabricate the nanocomposites. Solution-cast materials display no microscopic anisotropy, while samples that were cast and subsequently compression molded contain both isotropic regions as well as domains of locally oriented whiskers. Polarized optical microscopy showed these regions to have dimensions in the hundreds of $\mu \mathrm{m}$. Polarized Raman spectroscopy of the $1095 \mathrm{~cm}^{-1}$ Raman band, associated with $\mathrm{C}-\mathrm{O}$ ring stretching of the cellulose backbone, was used to quantify the local orientation of the cellulose whiskers. Clear and discernible shifts of this Raman band upon uniaxial deformation of nanocomposite films were further used to determine the level of stress experienced by the cellulose whiskers, ultimately reflecting the levels of stress-transfer predominantly between the poly(vinyl acetate) matrix and the tunicate whiskers, but also between the whiskers within the network. In the isotropic regions, where whiskers form a percolating network, the observed Raman shift rate with respect to strain is smaller than in the regions where the whiskers are uniaxially orientated. The Raman shift is strongly affected by the presence of water, leading to a lack of stress-transfer when the samples are fully hydrated, which is clearly detected by the Raman technique. Heating of the nanocomposites above the glass transition temperature of the poly(vinyl acetate) matrix also reduces the stress experienced by the individual whiskers.
\end{abstract}

\section{Introduction}

There is a need to better understand the nature of the interactions between cellulose nanofibers or "whiskers" and polymeric matrix materials due to the outstanding mechanical properties of the nanocomposites comprised of these components. These nanocomposites are attractive for many uses and are environmentally friendly as a consequence of the abundance of cellulosic biomass. ${ }^{1}$ Cellulose whiskers can be obtained from a range of renewable biosources, including wood, cotton, and sisal. A particularly interesting type of cellulose whisker can be isolated from a family of sea creatures called tunicates, the only type of animal that produces cellulose similar to plant materials. ${ }^{2}$ The whiskers obtained from these sea creatures exhibit high stiffness (tensile modulus $\sim 140 \mathrm{GPa}$ ) and dimensions at the nanometer scale (typically $26 \mathrm{~nm} \times 2.2 \mu \mathrm{m}$ ). ${ }^{3,4}$ The specific surface area and the average aspect ratio (length/ diameter) of these whiskers have been reported to be close to $170 \mathrm{~m}^{2} \mathrm{~g}^{-1}$ and $70-85$, respectively. ${ }^{3,4}$ Investigations of cellulose whiskers extracted from tunicates have also shown that they are highly crystalline and of a pure $\mathrm{I} \beta$ allomorph. ${ }^{5,6}$ The first study of a polymer nanocomposite comprising tunicate whiskers was reported by Favier et al. who combined these nanofibers with a polymer latex. ${ }^{7}$ The reinforcement was shown

* To whom correspondence should be addressed. Tel.: 01613065982 Fax: 0161306 3586. E-mail: s.j.eichhorn@manchester.ac.uk.

University of Manchester.

$\div$ Case Western Reserve University.

$\S$ University of Fribourg. to follow a percolation model, suggesting that the stress-transfer process is dominated by the formation of a continuous network of cellulose whiskers and strong interactions (hydrogen bonding) between them. ${ }^{7}$ Following this significant finding, many synthetic and natural polymers have been reinforced with cellulose whiskers. For example, Helbert et al. reported on the incorporation of cellulose whiskers isolated from wheat straw in a poly(styrene-co-butyl acrylate) latex. ${ }^{8}$ The whiskers were found to improve the thermal stability of the resulting composites at temperatures higher than the glass transition temperature $\left(T_{\mathrm{g}}\right)$ of the matrix, and the modulus of the composites increased with the whisker content. ${ }^{8}$ A similar thermal stabilization effect was reported when tunicate whiskers were incorporated into poly(ethylene oxide). ${ }^{9}$ This was again thought to be due to the formation of a rigid percolating cellulose whisker network via strong hydrogen bond interactions between the whiskers. ${ }^{9}$ Ljungberg et al. investigated the effect of the surface modification of tunicate whiskers on the mechanical properties of nanocomposites produced by solvent casting an isotactic polypropylene matrix. ${ }^{10}$ The modulus of these composites was found to be strongly dependent on the level of dispersion of the whiskers. ${ }^{10}$ Some of us have recently shown that the introduction of whiskers isolated from tunicates, microcrystalline cellulose, and cotton give rise to significant reinforcement of several polymers, including ethylene oxide/epichlorohydrin copolymers, polystyrene, polybutadiene, and poly(vinyl acetate). ${ }^{4,11}$ It was also demonstrated that the reinforcement effect of the whisker-whisker interaction could be "switched-off" by ex- 
posure of such materials to water. ${ }^{4,12}$ This effect was explained by a mechanism involving competitive hydrogen bonding of water with the whiskers, which reduces the whisker-whisker (and potentially whisker-matrix) interactions, and dramatically weakens the percolating whisker network in the polymer matrix. ${ }^{4,12}$ However, no direct spectroscopic evidence for this mechanism has yet been reported to the knowledge of the authors.

Raman spectroscopy has been used extensively to determine the micromechanical properties of polymer (nano)fibers and (nano)composites thereof. ${ }^{13}$ The technique relies on the change of the bond force constant of a bond in the backbone of the polymer molecules oriented along the (nano)fiber axis upon deformation of the fiber. This, in turn, results in a shift in the position of the Raman band associated with this structural moiety. Mitra et al. were the first to report such shifts for a deformed monocrystalline polydiacetylene fiber. ${ }^{14}$ Galiotis et al. determined the local axial strain in a polydiacetylene fiber embedded in an epoxy resin, which was the first time that the stress-transfer process in a composite had been directly monitored with this spectroscopic technique. ${ }^{15}$ Some of us have used Raman spectroscopy to determine the molecular deformation in several regenerated and natural cellulose fibers, ${ }^{16,17}$ and were able to adapt this technique to natural nanoscale whiskers and crystals of cellulose. Sturcova et al. reported the deformation of tunicate cellulose whiskers embedded in an epoxy resin based nanocomposite. ${ }^{18}$ The observed rate shift (with respect to $\%$ strain) of the Raman band located initially at $1095 \mathrm{~cm}^{-1}$ was $-2.4 \mathrm{~cm}^{-1} \%^{-1}$. $^{18}$ This value, through comparison to the rate shifts of other cellulose fibers, was interpreted to indicate efficient stress-transfer between whiskers and whiskers and the glassy matrix material. ${ }^{18}$ By interpolation of this shift rate, the modulus of the tunicate whiskers was estimated to be about $143 \mathrm{GPa}^{18}$ This value is in close agreement with a recent measurement of the bending stiffness of tunicate cellulose whiskers, obtained using an AFM (atomic force microscope) technique. ${ }^{19}$ Values of $145 \pm 31$ and $151 \pm 29 \mathrm{GPa}$ were determined for tunicate whiskers whose surface was modified by oxidation with 2,2,6,6-tetramethylpiperiditie-1-oxyl radical (TEMPO) and acid-hydrolyzed tunicate whiskers, respectively. ${ }^{19}$ In subsequent work, the same Raman technique has been used to show that whiskers produced from acid-hydrolyzed cotton have moduli in the range $54-100 \mathrm{GPa}^{20}$ Despite these critical measurements of the stiffness of tunicate cellulose whiskers, there is, however, still little direct evidence for the stress-transfer mechanisms between cellulose whiskers and polymer matrix materials in nanocomposites. This paper summarizes our recent efforts to use Raman spectroscopy to develop quantitative insights into the stress-transfer mechanisms that can occur in tunicate cellulose whisker/poly(vinyl acetate) nanocomposites. The results also show that the extent of stress-transfer is influenced by local orientation of the whiskers, which in turn is governed by the processing conditions used to fabricate the nanocomposites. The stress-transfer is also shown to be effected by the environmental conditions (temperature, moisture). This Raman spectroscopic approach could therefore be a useful method for monitoring and ultimately controlling the interfacial properties of new adaptive cellulose-based composites for a variety of applications.

\section{Materials and Methods}

Sample Preparation. Tunicates (Styela clava) were collected from floating docks at Point View Marina (Narragansett, RI) and whiskers were isolated and freeze-dried according to the procedures developed by Favier et al., ${ }^{7}$ Yuan et al., ${ }^{21}$ and some of us. ${ }^{12 c, 22}$ The detailed protocol employed here is found in ref $12 \mathrm{c}$.

To produce films consisting of the neat cellulose whiskers only, the freeze-dried whiskers were dispersed in dimethyl formamide (DMF, extra dry, Acros Organics) at a concentration of $5 \mathrm{mg} \mathrm{mL}^{-1}$ by ultrasonicating at ambient temperature in an ultrasonic bath (Fisher Scientific FS60H) for $12-16 \mathrm{~h}$. A total of $5 \mathrm{~mL}$ of the dispersion was cast into a Teflon Petri dish (diameter $3 \mathrm{~cm}$ ), and the dish was placed in a vacuum oven at $65^{\circ} \mathrm{C}$ and $-80 \mathrm{KPa}$ for 7 days. The resulting films had a thickness of $100-200 \mu \mathrm{m}$.

To produce cellulose whisker/poly(vinyl acetate) nanocomposites, $5 \mathrm{mg} \mathrm{mL}^{-1}$ whisker dispersions in DMF were prepared as described above. Poly(vinyl acetate) (PVAc, Sigma Aldrich, $M_{\mathrm{w}} \sim 113000 \mathrm{~g}$ $\mathrm{mol}^{-1}$ ) was dissolved in DMF (5\% w/w) by stirring overnight. A $12.2 \%$ $\mathrm{v} / \mathrm{v}$ whisker nanocomposite was prepared by combining $15 \mathrm{~mL}$ of the colloidal whisker dispersion and $8.5 \mathrm{~mL}$ of polymer solution, sonicating in the bath sonicator for $10 \mathrm{~min}$ and casting the homogeneous mixtures into Teflon Petri dishes (diameter $6.2 \mathrm{~cm}$ ). The dishes were placed into a vacuum oven $\left(65^{\circ} \mathrm{C},-80 \mathrm{KPa}, 1\right.$ week) to evaporate the solvent and dry the resulting films, which had a thickness of 100-200 $\mu \mathrm{m}$. Some of the resulting films were compression molded in a Carver laboratory press $\left(90{ }^{\circ} \mathrm{C}\right.$ at 0 psi for $2 \mathrm{~min}$, followed by a pressure of $3000 \mathrm{psi}$ for $15 \mathrm{~min}$ ) to yield $200-300 \mu \mathrm{m}$ thickness nanocomposite films. Some films were produced without this pressing step.

Scanning Electron Microscopy (SEM). Neat cellulose whisker films were sputter-coated with gold, using an Edwards S150B unit, to render them electrically conductive. The coated samples were examined in a field emission gun scanning electron microscope (FEG SEM; Philips XL30) operated at $5 \mathrm{kV}$.

Molecular Deformation Studies Using Raman Spectroscopy. A $26 \mathrm{~mW}$ (attenuated to $1 \mathrm{~mW}$ at the sample surface) $785 \mathrm{~nm}$ near-infrared laser coupled to a Renishaw System 1000 Raman spectrometer was used to record the spectra. A $50 \times$ objective lens with 0.60 numerical aperture was used to focus the laser beam onto the sample to a spot size of $2 \mu \mathrm{m}$. Raman spectra were acquired in the range of 1050 to $1150 \mathrm{~cm}^{-1}$, which includes the $1095 \mathrm{~cm}^{-1}$ cellulose ring-stretching mode. ${ }^{23}$ To obtain spectra with minimal noise, an exposure time of $30 \mathrm{~s}$ was used. Four spectra were accumulated and averaged, giving a total exposure time of $120 \mathrm{~s}$.

Thin films of the neat cellulose whiskers and the tunicate whisker/ PVAc nanocomposites (original sample length $=10 \mathrm{~mm}$ ) were incrementally deformed using a customized uniaxial tensile deformation rig with an attached load cell and strain (defined as the change in length of specimen divided by the original length) increments of $0.1 \%$. Raman spectra were recorded in situ after each deformation step. Samples were mounted on cardboard window frames, before attaching them to the platens of the deformation rig. The recorded Raman spectra were fitted using a mixed Gaussian/Lorentzian function, and an algorithm based on the work of Marquardt ${ }^{24}$ to determine the peak positions as a function of strain. The arrangement of laser and analyzer was such that their polarization direction was parallel to the long axis of the samples and, therefore, also to the tensile deformation axis of the specimens.

The above experiments were also conducted with samples that had been exposed to water or were exposed (in situ) to a temperature above the glass transition temperature $\left(T_{\mathrm{g}}\right)$. For the former, $12.2 \% \mathrm{v} / \mathrm{v}$ tunicate whisker/PVAc nanocomposites were cut into thin strips with dimensions of $10 \times 1 \times 0.2 \mathrm{~mm}$ and were immersed in deionized water at ambient temperature for 7 days. The samples were then attached to a cardboard window frame before being secured to the platens of the tensile rig. The samples were kept wet during the deformation experiments by wetting them with droplets of deionized water. To probe the effect of temperature, the samples were attached to the tensile rig as described for the wet samples. A temperature-controlled heat source that was suspended approximately $20 \mathrm{~mm}$ below the sample was found to be suitable for locally heating the samples, while the deformation experiments were conducted. The temperature was monitored with a thermocouple-based digital thermometer and was maintained at ap- 


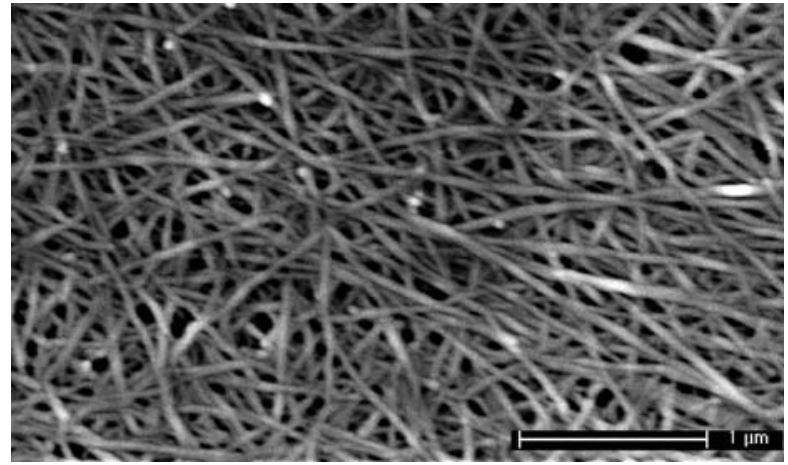

Figure 1. Typical scanning electron microscope (SEM) image of a neat tunicate whisker film produced by solution-casting a $5 \mathrm{mg} \mathrm{mL}^{-1}$ whisker dispersion in DMF.

proximately $60{ }^{\circ} \mathrm{C}$, which is above the glass transition temperature of the PVAc matrix.

\section{Results and Discussion}

Sample Preparation and Mechanical Properties. The samples studied here are the mechanically adaptive nanocomposites based on poly(vinyl acetate) (PVAc) and tunicate whiskers reported before. ${ }^{12}$ The cellulose whiskers employed were isolated from the species Styela clava, as reported in detail elsewhere, ${ }^{12 \mathrm{c}}$ they had a typical diameter of about $20 \mathrm{~nm}$, an average length of $2.2 \mu \mathrm{m}$ and an average aspect ratio of $\sim 84$. The hydrolysis of the tunicate mantles with sulfuric acid introduces a moderate concentration of surface sulfate groups $\left(\sim 85 \mathrm{mmol} \mathrm{kg}^{-1}\right)$, which cause electrostatic repulsion between the whiskers and are responsible for good dispersibility in polar solvents. ${ }^{22}$

Figure 1 shows a typical scanning electron microscopy (SEM) image of a neat tunicate cellulose whisker film that was produced by solution-casting from dimethylformamide (DMF) and subsequent drying. The image clearly reveals the formation of a dense, percolating whisker network. Similar network structures (although less dense) were observed for tunicate whisker/polymer nanocomposites processed with the same protocol as the materials studied here. The tensile storage modulus $\left(E^{\prime}\right)$ of these neat whisker films, measured by dynamic mechanical analysis (DMA), ranges from $8-8.5 \mathrm{GPa}\left(\right.$ at $25^{\circ} \mathrm{C}$ ).

Nanocomposite films based on poly(vinyl acetate) (PVAc) and $12.2 \% \mathrm{v} / \mathrm{v}$ tunicate whiskers were also produced by solution casting from DMF. Optionally, these materials were subsequently reshaped by compression molding (see Materials and Methods for details). The two processing methods resulted in materials with identical mechanical properties at $25^{\circ} \mathrm{C} ; E^{\prime}$ was in both cases $\sim 5 \mathrm{GPa}$. Above the glass transition, the compression molded material displayed a slightly lower modulus than the cast sample (at $80{ }^{\circ} \mathrm{C}, E^{\prime}=700 \mathrm{MPa}$ and $1.1 \mathrm{GPa}$, respectively; see Supporting Information and DMA data in Figure 1a), perhaps on account of lower crystallinity. As discussed in an earlier study, ${ }^{12}$ the whisker/PVAc nanocomposites display chemo-responsive mechanical properties; the modulus is significantly reduced upon immersion in water. After immersion in water at room temperature for one week, $E^{\prime}$ of the compression-molded sample was found to drop to $\sim 36 \mathrm{MPa}$ (at $25^{\circ} \mathrm{C}$ ). At this temperature, the neat dry PVAc has an $E^{\prime}$ of $\sim 2 \mathrm{GPa}$, which drops to $4 \mathrm{MPa}$ upon immersion in water.

Raman Spectroscopy and Molecular Deformation. Previous research ${ }^{16-18}$ has shown that natural cellulose fibers have a well-defined Raman peak at $1095 \mathrm{~cm}^{-1}$, which is associated

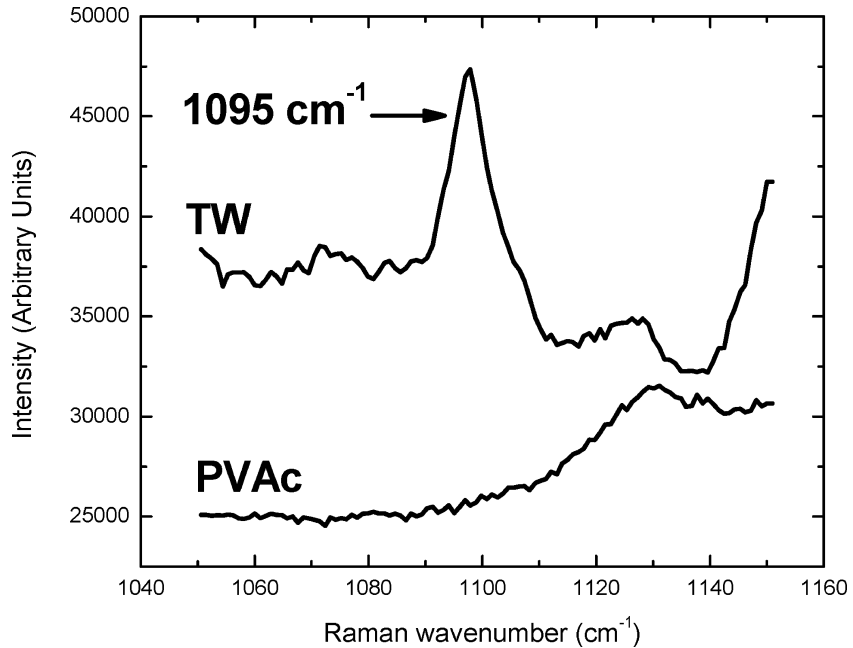

Figure 2. Typical Raman spectra of films of neat tunicate whiskers (TW) and neat poly(vinyl acetate) (PVAc) polymer. The position of the $1095 \mathrm{~cm}^{-1}$ peak associated with $\mathrm{C}-\mathrm{O}$ ring stretching of the cellulose backbone is highlighted with an arrow.

with $\mathrm{C}-\mathrm{O}$ ring stretching. ${ }^{23}$ Therefore, only the relatively narrow region between 1050 and $1150 \mathrm{~cm}^{-1}$ was monitored in this study. The Raman spectra of films of neat tunicate whiskers and the neat PVAc matrix are shown in Figure 2. The Raman spectrum acquired for the neat tunicate whisker film shows, as expected, an intense peak located at approximately $1095 \mathrm{~cm}^{-1}$, while the spectrum of the neat PVAc matrix is devoid of any resonances in this region (Figure 2). It is therefore possible to use the position of this Raman peak to monitor the local strain state of cellulose whiskers in tunicate whisker/PVAc nanocomposites.

Tunicate whisker/PVAc nanocomposite films that were processed by solution casting only, and samples that were reshaped by compression molding (vide supra) were first examined by optical microscopy. Typical images of a compression-molded sample viewed in both unpolarized light and under crossed polarizers are shown in Figure 3a,b, respectively. In unpolarized light, the sample appears transparent, apart from a few surface asperities, possibly due to dust or whisker aggregates, which appear as dark regions (Figure 3a). When viewed between crossed polarizers, dark and bright regions with dimensions of the order of several hundred $\mu \mathrm{m}$ can be discerned (Figure 3b). Samples that were simply solution cast, showed no such local variation (Figure 3c), and so they are thought to contain isotropically oriented whiskers. Some dark regions in the polarized optical micrographs of compression-molded films remained dark if the sample was rotated, while the intensity of the bright areas changed significantly. This local birefringence is consistent with a high level of uniaxial orientation of large domains of whiskers, which is thought to be induced by shear flow during processing, and which, on account of the high viscosity of the nanocomposite, has not relaxed before the sample was cooled to below $T_{\mathrm{g}}$. The concentration of whiskers within each region (light and dark) is not known, and so it is difficult to determine whether nematic ordering, of a nature seen in dried films of pure cellulose nanowhiskers, ${ }^{25}$ has occurred. It is thought, however, that the dominating driving force must be pressing given that no ordered domains are seen in the solution cast material, which has a similar thickness to the pressed material. On the other hand, the dark regions in the polarized optical micrographs seem to represent areas in which the cellulose whiskers form a (presumably randomly oriented) whisker network. To further support this interpretation 
(a)

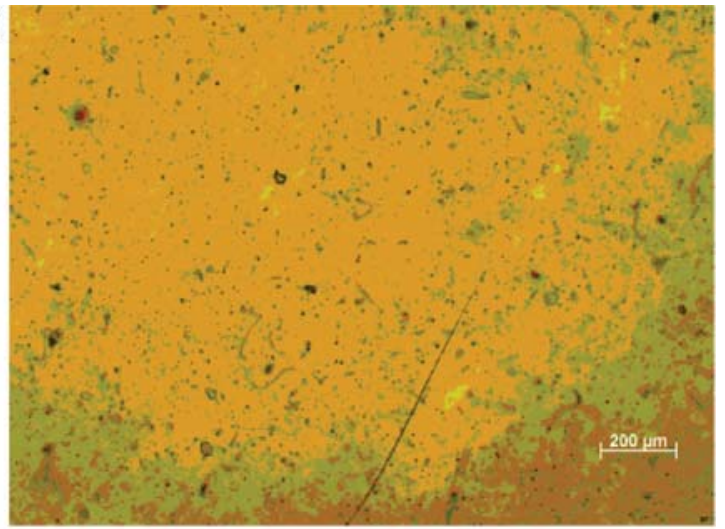

(b)

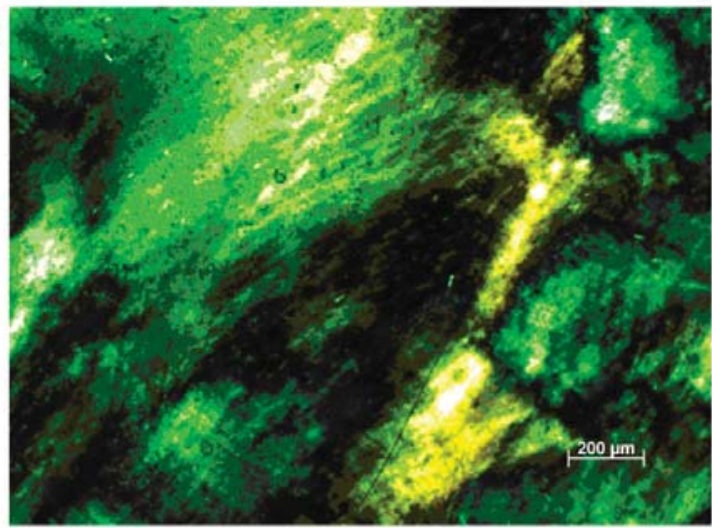

(c)

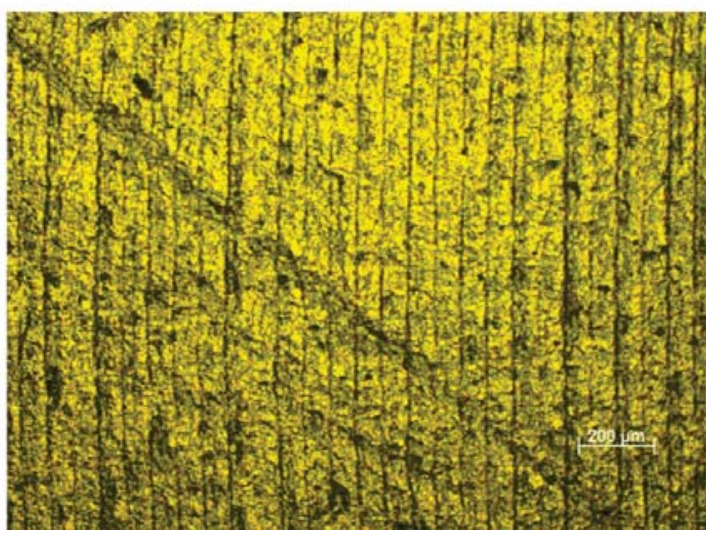

Figure 3. Typical optical microscopy images of (a) a $12.2 \% \mathrm{v} / \mathrm{v}$ tunicate whisker/poly(vinyl acetate) (TW/PVAc) nanocomposite film that was produced by solution-casting from DMF and subsequent compression-molding at $90^{\circ} \mathrm{C}$; (b) same as in (a), but viewed under crossed polarizers; (c) a $12.2 \% \mathrm{v} / \mathrm{v}$ TW/PVAc nanocomposite film that was produced by solution-casting from DMF viewed under crossed polarizers.

and to possibly discriminate between the stress-transfer properties of the presumably randomly oriented whisker network structures and regions of uniaxially oriented whiskers, we used polarized optical microscopy to locate such areas on the sample before conducting localized Raman experiments. The laser and the analyzer of the Raman spectrometer were arranged with their polarization directions parallel to one another, with the deformation axis of the samples parallel to this polarization axis. The sample was then rotated in $5^{\circ}$ intervals around $360^{\circ}$; the intensity of the Raman band located at $1095 \mathrm{~cm}^{-1}$ was recorded at each position, and a polar plot was constructed. Figure 4a shows the Raman data of a $12.2 \%$ v/v tunicate whisker/poly(vinyl acetate) nanocomposite film that was produced by solution-casting from DMF, and subsequently compression-molded at $90{ }^{\circ} \mathrm{C}$, in an (a)

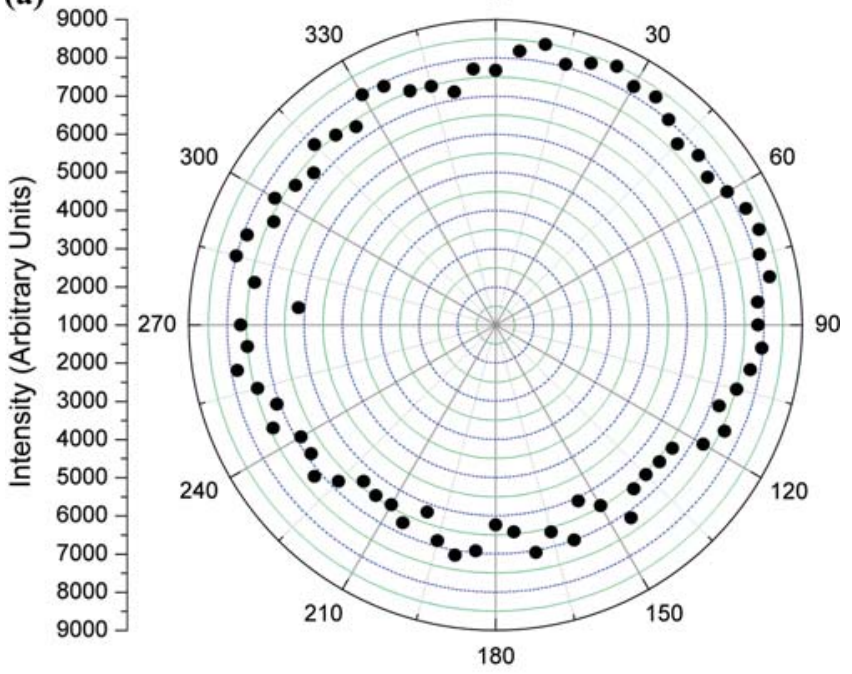

(b)

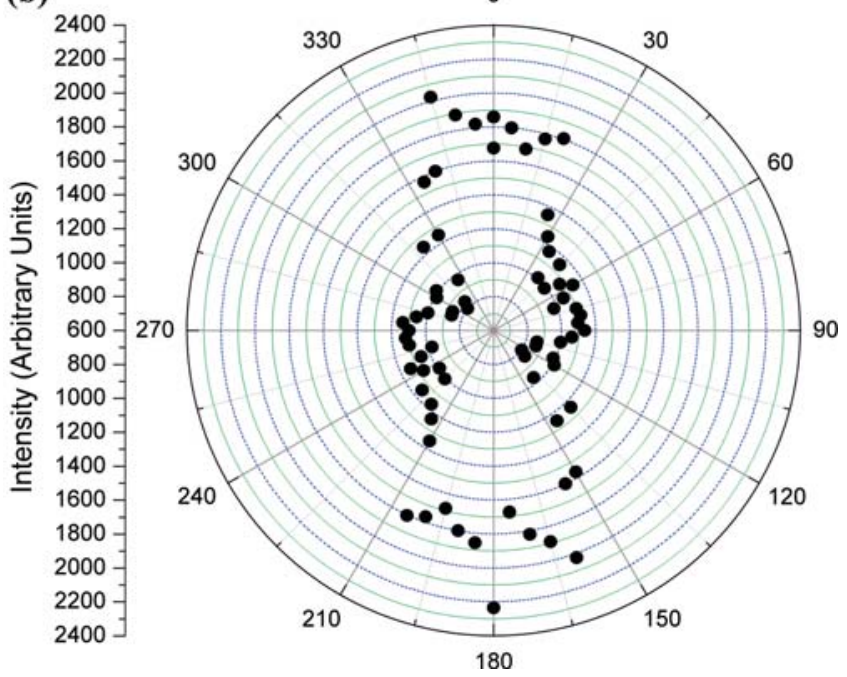

Figure 4. Plots showing the intensity of the $1095 \mathrm{~cm}^{-1}$ Raman band as a function of rotation angle (polar scale) of a $12.2 \% \mathrm{v} / \mathrm{v}$ tunicate whisker/poly(vinyl acetate) (TW/PVAc) nanocomposite film that that was produced by solution-casting from DMF and subsequent compression-molding at $90^{\circ} \mathrm{C}$. (a) Data acquired in the dark region of the specimen shown in Figure 3b. (b) Data acquired in the bright region of the specimen shown in Figure $3 \mathrm{~b}$. The zero degrees line is parallel to the long axis of the specimen.

area that was identified as dark by polarized optical microscopy. It can be seen that these data also support the notion that in this region the cellulose whiskers adopt a random orientation; no intensity maximum is observed in any direction. Similar orientation distributions were obtained for samples that were simply solution cast, without the pressing stage (see Supporting Information, Figure 1b). The corresponding data recorded in an area that was identified as bright by polarized optical microscopy, shown in Figure $4 \mathrm{~b}$, paint a different picture. In this case, a significant local anisotropy is found, as seen by the strong polarization of the Raman band located at $1095 \mathrm{~cm}^{-1}$. Because this band is related to the $\mathrm{C}-\mathrm{O}$ stretch along the chain axis of the cellulose molecules, ${ }^{23}$ which in turn are thought to be oriented along the longitudinal axis of the whiskers, this result points to a significant level of local uniaxial orientation. Orientation of the whiskers is thought to result from flow of the viscous polymer matrix during melt processing. The variations of local whisker orientation, which were observed here for the first time, and which are apparently the result of 


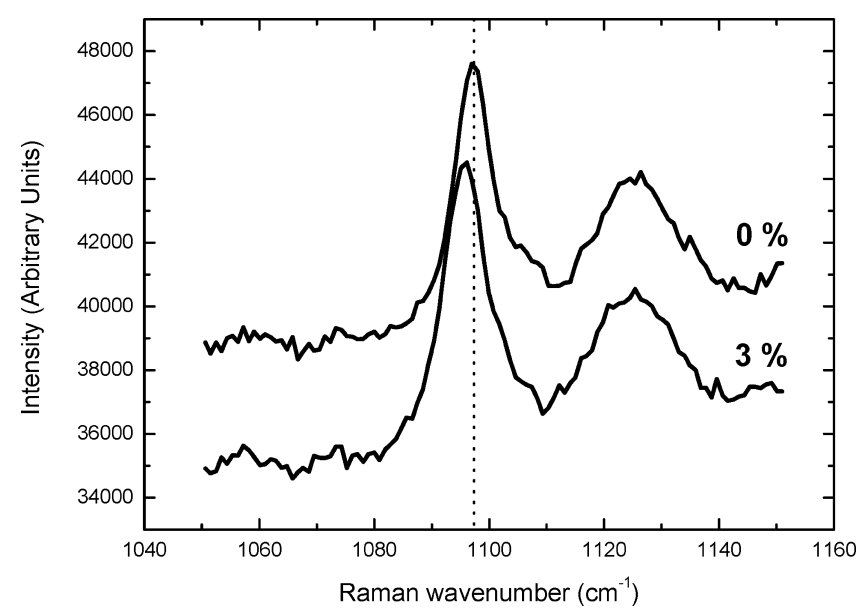

Figure 5. Raman spectra of a $12.2 \% \mathrm{v} / \mathrm{v}$ tunicate whisker/poly(vinyl acetate) (TW/PVAc) nanocomposite film that was produced by solution-casting from DMF and subsequent compression-molding at $90{ }^{\circ} \mathrm{C}$. Shown is the region around the Raman band located at 1095 $\mathrm{cm}^{-1}$ before $(0 \%)$ and upon application of uniaxial strain (3\%). The dotted line indicates the initial position of the band.

the specific process employed to fabricate these materials, are interesting and potentially of importance for the stress-transfer mechanisms in these materials. The ease with which apparently a high level of anisotropy can be introduced, and the slow relaxation may be exploited to create macroscopically oriented whisker nanocomposites.

Stress-Transfer Mechanisms in Neat Cellulose Whisker Films and Nanocomposites. The typical change in the position of the Raman peak located at $1095 \mathrm{~cm}^{-1}$ that is observed upon uniaxially stretching (3\% strain) a $12.2 \% \mathrm{v} / \mathrm{v}$ tunicate whisker/ PVAc nanocomposite film (that was produced by compression molding) is shown in Figure 5. The observed shift in the position of this peak has been shown to be related to the deformation of the backbone of individual cellulose molecules ${ }^{16-18}$ because a change in the stress-state of the molecular chain results in a shift in the vibrational frequency due to an alteration of the force constant. ${ }^{13,14}$ In a neat cellulose whisker film in which the cellulose whiskers form a dense network, stress-transfer through the material relies completely on strong, noncovalent whisker-whisker interactions. In the nanocomposites, whiskerwhisker interactions are also thought to be present, but matrix-whisker stress-transfer can also be assumed to take place. In fact, in our previous investigations, ${ }^{4,12}$ we elucidated this aspect in the framework of two classical models, namely, the percolation model ${ }^{26}$ and the Halpin-Kardos model. ${ }^{27}$ The percolation model predicts the modulus of a nanocomposite that comprises a percolating filler network, where the matrix is too soft to contribute to the overall modulus and the filler network, which relies on strong filler-filler interactions, dominates the overall stiffness. The Halpin-Kardos model on the other hand assumes that the filler particles are well dispersed in the matrix without any interactions between them. We have shown before that the modulus of dry whisker/PVAc nanocomposites above $T_{\mathrm{g}}$ agrees with the percolation model, while the Halpin-Kardos equations adequately describe the modulus of water-swollen nanocomposites, suggesting that the filler-filler interactions are "switched off" in the latter, by way of competitive hydrogen bonding with water.

Using a custom-built setup that allowed the highly localized acquisition of Raman spectra in situ, while conducting a uniaxial stress-strain experiment (while optionally also exposing the samples to water or high temperature, vide infra), we monitored

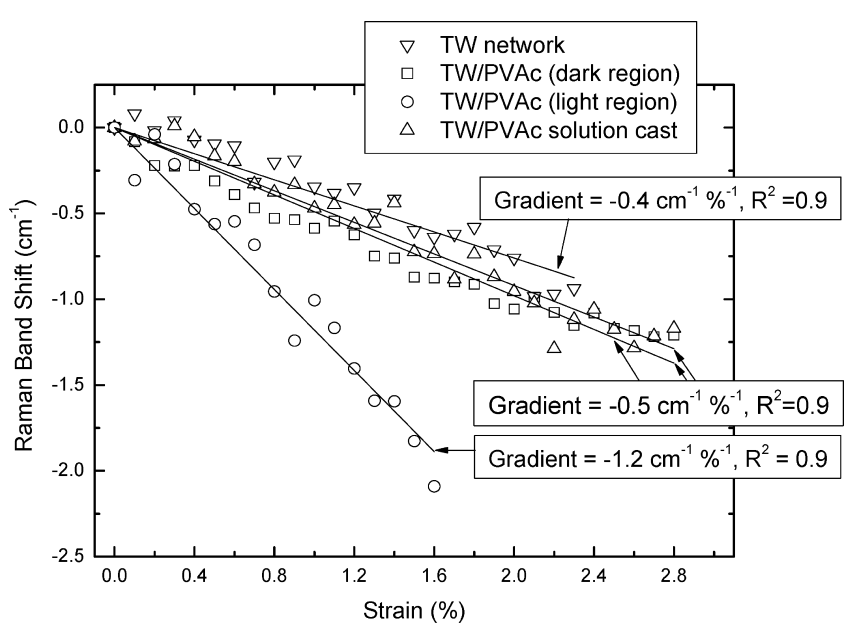

Figure 6. Typical shifts of the position of the Raman band located at $1095 \mathrm{~cm}^{-1}$ as a function of uniaxial strain for a solution-cast neat tunicate whisker film (TW), and for a $12.2 \% \mathrm{v} / \mathrm{v}$ tunicate whisker/ poly(vinyl acetate) (TW/PVAc) nanocomposite film that was produced by solution-casting from DMF and subsequent compression-molding at $90{ }^{\circ} \mathrm{C}$. For the latter sample, data were acquired in both dark and bright regions of the specimen shown in Figure $3 \mathrm{~b}$.

the position of the Raman band located at $1095 \mathrm{~cm}^{-1}$ as a function of tensile deformation for all samples under investigation. In all cases, the position of this Raman band shifts linearly toward a lower wavenumber as shown in Figure 6. Employing linear regression analysis, the rates of the Raman band shifts with respect to strain were elucidated. The shift rate was determined to be $-0.4 \mathrm{~cm}^{-1} \%^{-1}$ for a solution-cast film consisting of neat cellulose whiskers; similar values $\left(-0.5 \mathrm{~cm}^{-1}\right.$ $\%^{-1}$ ) were found for solution-cast films of the $12.2 \% \mathrm{v} / \mathrm{v}$ tunicate whisker/PVAc nanocomposite and the dark regions in the polarized optical micrographs of a compression-molded film of the $12.2 \% \mathrm{v} / \mathrm{v}$ tunicate whisker/PVAc nanocomposite. On the other hand, measurements in the bright regions in the polarized optical micrographs of a compression-molded film of the $12.2 \%$ v/v tunicate whisker/PVAc nanocomposite yielded a shift rate of $-1.2 \mathrm{~cm}^{-1} \%^{-1}$. It must be noted that the scatter in the position of the Raman band located at $1095 \mathrm{~cm}^{-1}$ from an unstrained sample rarely exceeds $\pm 0.2 \mathrm{~cm}^{-1}$, suggesting that the response of the bright regions in the polarized optical micrographs of a compression-molded film of the $12.2 \% \mathrm{v} / \mathrm{v}$ tunicate whisker/PVAc nanocomposite is indeed significantly different from that of the other samples. It must also be noted that some local density variations will occur in the samples and, like most conventional fiber composites, it is likely that higher volume fractions or better packing of whiskers can be achieved in the oriented regions. This will affect the local stress levels within the composite to some extent. However, our data suggests that whisker-whisker interactions (hydrogen bonding) among randomly oriented whiskers, which form a percolating network, dominate the stress-transfer process in neat whisker films, solution-cast whisker/PVAc nanocomposites, and the isotropic regions of compression-molded whisker/PVAc nanocomposites. However, in the composite materials, the stress-transfer between resin and whiskers also plays a significant role in the mechanics of the materials. The significantly higher shift rate in the anisotropic local domains of compression-molded tunicate whisker/PVAc nanocomposite is an indication of the dominance of matrix-whisker interactions and a greater level of axial deformation of the whiskers, when they are oriented with respect to the tensile axis of the specimen. Greater axial stiffness of cellulose nanocomposites could therefore be achieved via 

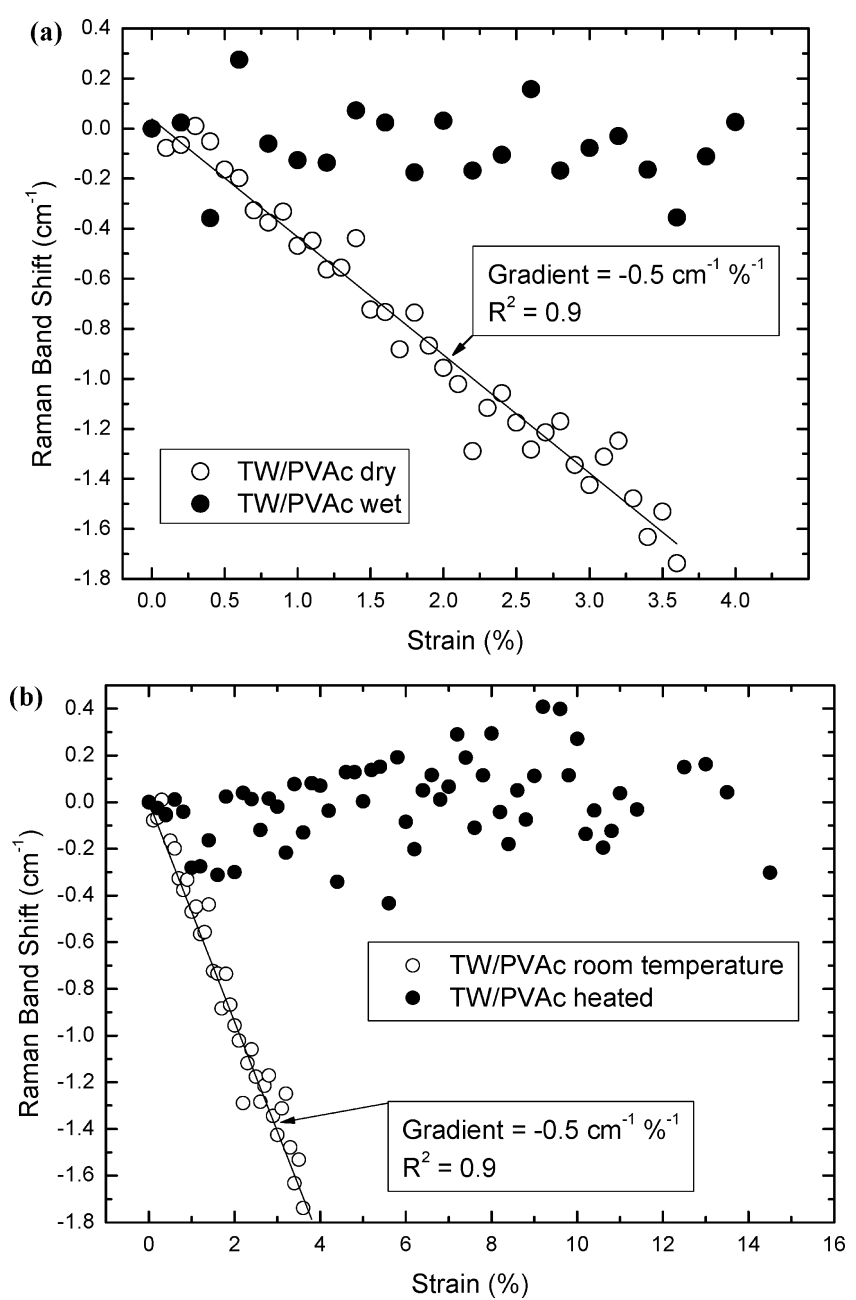

Figure 7. Typical shifts of the position of the Raman band located at $1095 \mathrm{~cm}^{-1}$ as a function of uniaxial strain for a $12.2 \% \mathrm{v} / \mathrm{v}$ tunicate whisker/poly(vinyl acetate) (TW/PVAc) nanocomposite film that was produced by solution-casting from DMF and subsequent compressionmolding at $90{ }^{\circ} \mathrm{C}$ as function of exposure to water (a: the sample was kept wet during deformation) and temperature (b: the sample was heated in situ to $60^{\circ} \mathrm{C}$ ). The data of the original film (cf. Figure $6 \mathrm{~b}$; dark region of the specimen shown in Figure $3 \mathrm{~b}$ ) are included for comparison.

orientation of the whisker component. It should be pointed out that the shifts in the Raman peaks are as a consequence of the stress-transfer between the resin and the whiskers and, also, in the dark regions of the sample, between whiskers.

To develop a better understanding for the stress-transfer mechanisms that occur in cellulose whisker based nanocomposites, in particular the role of whisker-whisker and whiskermatrix interactions in the nanocomposites under investigation, we monitored the molecular deformation of the cellulose whisker component in the absence and presence of water, which was shown before to slightly swell the tunicate whisker/PVAc nanocomposites, plasticize the matrix and (by way of competitive hydrogen bonding with the whiskers) reduce the whiskerwhisker interactions and therewith the overall stiffness of the materials. ${ }^{4,12}$ A comparison of the shifts of the position of the Raman band located at $1095 \mathrm{~cm}^{-1}$ upon uniaxial tensile deformation of wet and dry tunicate whisker/PVAc nanocomposite films (acquired in dark regions in the polarized optical micrographs of a compression-molded film of the $12.2 \% \mathrm{v} / \mathrm{v}$ tunicate whisker/PVAc nanocomposite) is presented in Figure 7a. Interestingly, the Raman band shift rate of $-0.5 \mathrm{~cm}^{-1} / \%$ for a dry sample (vide supra) is reduced to zero (no observable band shift) if the sample is immersed in deionized water to equilibrium. It has been reported earlier that the matrix plasticizes when submerged in water. This suggests that the stress-transfer between the PVAc matrix and the cellulose whiskers, and between the whiskers, have been reduced to a point where it has become undetectable using the Raman technique. In fact, one can correlate this with the drastic change in macroscopic mechanical behavior of these nanocomposites (specifically the tensile storage modulus) that was observed in our previous investigations upon exposing these samples to water (vide supra). ${ }^{4,12 a}$ In another study, de Rodriguez et al. ${ }^{28}$ reported the water uptake activity in sisal whisker/PVAc composite films. Absorbed water molecules were reported to accumulate in three regions within the cellulose nanocomposites, namely, within the PVAc matrix, between whiskers, and at the polymer-whisker interface. ${ }^{29}$ However, crystalline cellulose does not absorb a significant amount of water, ${ }^{29}$ and neat PVAc by itself is not very hygroscopic (water uptake below $4 \mathrm{wt}$ $\% / w t),{ }^{4,12 a}$ which implies that at equilibrium the polymerwhisker interface region accommodates most of the absorbed water molecules, concomitant with a breakdown of both the whisker-whisker and whisker-matrix interactions due to competitive hydrogen bonding with water. We measured the water content of the samples after wetting and found this to be $27.8 \pm 1.5 \mathrm{wt} \%$ (based on the dry weight of the samples and for a $15 \mathrm{wt} \%$ of whiskers). This suggests that indeed the moisture is accommodated at the interface between the polymer and the whiskers, assuming that little moisture is absorbed by the whiskers themselves given their presumably high crystallinity.

Because we recorded stress levels on our samples during all deformation studies using the Raman spectrometer, we were able to subsequently plot stress-strain data for each of our materials (wet, dry (pressed), and dry (nonpressed)). These data are contained in Supporting Information (Figure 1c). It is noted that the wet samples show negligible stress levels compared to the dry material, which shows how dramatic the effect of moisture is on the mechanical properties of the composite when the whisker-whisker interface is disrupted. The pressed samples are initially stiffer than the nonpressed material, presumably due to the presence of anisotropic regions in the sample. However, the data eventually converge for these two samples, suggesting the whisker orientation occurs in the isotropic nonpressed material.

Differential scanning calorimetry (DSC) measurements (methods in Supporting Information) show that the glass transition temperature of the whisker/PVAc nanocomposite is $44^{\circ} \mathrm{C}$. The reduction of the tensile storage modulus of the $12.2 \% \mathrm{v} / \mathrm{v}$ TW/ PVAc nanocomposite upon heating above $T_{\mathrm{g}}$ (from $5 \mathrm{GPa}$ below $T_{\mathrm{g}}$, to about $700 \mathrm{MPa}$ above) is comparably small to that of the neat PVAc (from $2 \mathrm{GPa}$ to $4 \mathrm{MPa}$ ), ${ }^{4,12 a}$ consistent with dominant whisker-whisker stress-transfer above $T_{\mathrm{g}}$. This interpretation is now further supported by Raman experiments at $60{ }^{\circ} \mathrm{C}$. These data show that there is no significant shift of the Raman band located at $1095 \mathrm{~cm}^{-1}$ upon deforming the whisker/PVAc nanocomposites above $T_{\mathrm{g}}$ (Figure $7 \mathrm{~b}$ ). We observed some scatter in these data $\left( \pm 0.4 \mathrm{~cm}^{-1}\right)$, but this is much less than the shift in the peak when the sample is below $T_{\mathrm{g}}\left(>1.6 \mathrm{~cm}^{-1}\right)$. Because the Raman band shifts in cellulose are known to be stress induced, ${ }^{13,17}$ this indicates that stress-transfer from the matrix to the cellulose whiskers is reduced considerably above the glass transition. It is therefore thought that the rate of band shift is an indication of the breakdown of the matrix-whisker interactions. 


\section{Conclusions}

Raman spectroscopy has been successfully utilized to quantify the stress-transfer mechanisms of tunicate whisker networks and tunicate whisker/PVAc composites. The Raman spectra from both samples are characterized by a strong peak located, prior to deformation, approximately at $1095 \mathrm{~cm}^{-1}$. The intensity of this peak has been shown to change in regions where the whiskers are oriented due to pressing of the sample and subsequent shear flow. Other regions of the same sample are shown to have whiskers present in a random network structure. This local anisotropy has been shown to have an effect on the local micromechanical properties of the nanocomposites. The position of the Raman band located at $1095 \mathrm{~cm}^{-1}$ has been monitored while deforming the samples in tension, in both regions of the sample where whiskers are thought to be oriented and where random networks exist. This Raman band corresponds to the vibration of bonds within the backbone of the cellulose molecule and is dominated by a $\mathrm{C}-\mathrm{O}$ stretching motion. It is shown that the position of this band shifts toward a lower wavenumber position upon the application of external tensile deformation of the samples, which is an indication of the level of stress-transfer from the polymer matrix to the cellulose whiskers. Gradients of a linear fit to the position of this band with respect to strain for networks of pure whiskers and the composite samples are found to be $-0.4 \mathrm{~cm}^{-1} \%^{-1}$, and either -0.5 or $-1.2 \mathrm{~cm}^{-1} \%^{-1}$ for regions of the specimen with random or oriented whiskers, respectively. It is shown that distinct band shift motifs are obtained from regions of the pressed samples where networks of whiskers and oriented whiskers occur. In solution cast samples, where no pressing is applied, the whiskers do not orient, and the band shift rate with respect to strain is found to be similar to pure networks of whiskers without the presence of resin. This shows that this technique can be used to better understand local anisotropy in cellulose nanocomposites, and the corresponding relationship with the stress-transfer process can be obtained. By wetting the samples, it has also been shown that that interface in these composites can be "switched-off". When this occurs, no discernible shift in the Raman band located at $1095 \mathrm{~cm}^{-1}$ occurs, which is an indication of the breakdown of the matrix - whisker interaction and, therefore, a reduction in the stress-transfer between these two components. It is not possible to discriminate this from stress-transfer between whiskers. The samples were also heated above the glass transition temperature of the matrix material, which then became ductile. In this state the stresstransfer from the matrix to the whiskers is reduced, although whisker-whisker interactions are still thought to occur, leading to less of a reduction in mechanical properties than when the samples are wet.

Acknowledgment. The authors wish to thank the Ministry of Science, Technology and Innovation, Malaysia, for funding a Ph.D. studentship (to R.R.) and Case Western Reserve University for an Ohio Innovation Incentive Fellowship (to K.S.).

Supporting Information Available. The methods used for obtaining differential scanning calorimetry (DSC) and dynamic mechanical analysis (DMA) data are included in this section. DMA data for composite samples (pressed and solution cast) are also reported, as well as the orientation data for a solution cast sample and the stress-strain curves obtained from composites in both wet and dry states.

\section{References and Notes}

(1) Eichhorn, S. J.; Dufresne, A.; Aranguren, M.; Capadona, J. R.; Rowan, S. J.; Weder, C.; Thielemans, W.; Roman, M.; Renneckar, S.; Gindl, W.; Weigel, S.; Yano, H.; Abe, K.; Nogi, M.; Mangalam, A.; Simonsen, J.; Benight, A. D.; Bismarck, A.; Berglund, L. A. J. Mater. Sci. 2010, 45, 1 .

(2) Nishiyama, Y.; Langan, P.; Chanzy, H. Fiber Diffr. Rev. 2003, 11, $75-78$.

(3) (a) Azizi Samir, M. A. S.; Alloin, F.; Sanchez, J.; Dufresne, A. Polímeros 2005, 15, 109-113. (b) De Souza Lima, M.; Borsali, R. Macromol. Rapid Commun. 2004, 25, 771.

(4) Capadona, J. R.; Shanmuganathan, K.; Tyler, D. J.; Rowan, S. J.; Weder, C. Science 2008, 319, 1370-1374.

(5) Belton, P. S.; Tanner, S. F.; Cartier, N.; Chanzy, H. Macromolecules 1989, 22, 1615-1617.

(6) Kimura, S.; Itoh, T. In Biogenesis and Function of Cellulose in the Tunicates in Cellulose: Molecular and Structural Biology; Brown, R. M., Nobles D. R., Eds.; Springer: New York, 2007; pp 217-236.

(7) Favier, V.; Chanzy, H.; Cavaillé, J. Y. Macromolecules 1995, 28, 6365-6367.

(8) Helbert, W.; Cavaillé, J. Y.; Dufresne, A. Polym. Compos. 1996, 17, 604-611.

(9) Azizi Samir, M. A. S.; Alloin, F.; Sanchez, J. Y.; Dufresne, A. Polymer 2004, 45, 4149-4157.

(10) Ljungberg, N.; Cavaillé, J. Y.; Heux, L. Polymer 2006, 47, 62856292.

(11) (a) Capadona, J. R.; van den Berg, O.; Capadona, L.; Schroeter, M.; Tyler, D.; Rowan, S. J.; Weder, C. Nat. Nanotechnol. 2007, 2, 765. (b) Capadona, J. R.; Shanmuganathan, K.; Trittschuh, S.; Seidel, S.; Rowan, S. J.; Weder, C. Biomacromolecules 2009, 10, 712-716.

(12) (a) Shanmuganathan, K.; Capadona, J. R.; Rowan, S. J.; Weder, C. Prog. Polym. Sci. 2010, 35, 212-222. (b) Shanmuganathan, K.; Capadona, J. R.; Rowan, S. J.; Weder, C. J. Mater. Chem. 2010, 20, 180-186. (c) Shanmuganathan, K.; Capadona, J. R.; Rowan, S. J.; Weder, C. ACS Appl. Mater. Interfaces 2010, 2, 165-174.

(13) Young, R. J.; Eichhorn, S. J. Polymer 2007, 48, 2-18.

(14) Mitra, V. K.; Risen, W. M.; Baughman, R. H. J. Chem. Phys. 1977, 66, 2731-2736

(15) Galiotis, C.; Young, R. J.; Yeung, P. H. J.; Batchelder, D. N. J. Mater. Sci. 1984, 19, 3640-3648.

(16) Hamad, W. Y.; Eichhorn, S. J. J. Eng. Mater. Technol. 1997, 119, 309-313.

(17) Eichhorn, S. J.; Sirichaisit, J.; Young, R. J. J. Mater. Sci. 2001, 36, 3129-3135.

(18) Sturcova, A.; Davies, G. R.; Eichhorn, S. J. Biomacromolecules 2005, 6, 1055-1061.

(19) Iwamoto, S.; Kai, W. H.; Isogai, A.; Iwata, T. Biomacromolecules 2009, 10, 2571-2576.

(20) Rusli, R.; Eichhorn, S. J. Appl. Phys. Lett. 2008, 93, 033111.

(21) Yuan, H.; Nishiyama, Y.; Wada, M.; Kuga, S. Biomacromolecules 2006, 7, 696-700.

(22) van der Berg, O.; Capadona, J. R.; Weder, C. Biomacromolecules 2007, $8,1353-1357$.

(23) Wiley, J. H.; Atalla, R. H. Carbohydr. Res. 1987, 160, 113-129.

(24) Marquardt, D. W. J. Soc. Ind. Appl. Math. 1963, 11, 431-441.

(25) Revol, J. F.; Godbout, L.; Gray, D. G. J. Pulp Pap. Sci. 1998, 24, 146-149.

(26) Takayanagi, M.; Uemura, S.; Minami, S. J. Polym. Sci., Part C 1964, $5,113-122$

(27) Halpin, J. C.; Kardos, J. L. J. Appl. Phys. 1972, 43, 2235-2241.

(28) de Rodriguez, N. L. G.; Thielemans, W.; Dufresne, A. Cellulose 2006, $13,261-270$.

(29) Stromme, M.; Mihranyan, A.; Ek, R.; Niklasson, G. A. J. Phys. Chem. B 2003, 107, 14378-14382. 